\title{
Designing a Science-Based Strategy to Prepare for the Next Pandemic
}

\author{
Michelle Ma ${ }^{1}$, Reto Asmis" and Mark Crowder* \\ ${ }^{1}$ The Bryn Mawr School, Baltimore, MD, USA \\ \#Mentor
}

\section{$\underline{\text { ABSTRACT }}$}

This essay examines the consequences of the COVID-19 pandemic on the United States and recommends new and improved strategies for future pandemics accordingly. The purpose of this project is to explore pandemic measures taken by the United States administration and to analyze the efficacy of each. However, in order to better understand the nature of the virus, the essay also explores possible origins of the virus, its structure, and how it is transmitted. Statistics regarding the COVID-19 pandemic (i.e. the number of total deaths and infections) were then collected from various sources which include the $\mathrm{CDC}, \mathrm{NIH}$, and the WHO. After this data was analyzed, it was apparent that the exponential growth of new cases and deaths each day during the beginning of the pandemic could have been prevented by stricter protocols. In the event of a similar pandemic in the future, the recommended protocols in this essay are potential strategies to avoid another pandemic as deadly as the current COVID-19 pandemic.

\section{An Introduction to COVID-19 and an Examination of the Pandemic's Consequences on the United States}

The COVID-19 disease, caused by the SARS-CoV-2 virus, first infected a small cluster of people in Wuhan, China in late 2019 (CDC, 2020). Within months, the virus quickly made its way around the world, infecting people in almost all countries and resulting in over 4 million deaths globally, with over 600 thousand deaths just in the United States alone (McPhillips \& Gan, 2021). In the US, the exponential rise in new cases in early 2020 was a result of inadequate preparation and the rapid spread of misinformation (WHO, 2021). As a result, schools nationwide were shut down and moved online, affecting the quality of education for millions of students (UNESCO, 2021). Unfortunately, another pandemic similar to that of COVID-19 is inevitable in the future. Thus, it is imperative that the country is adequately prepared for the next pandemic in order to avoid such devastating consequences.

The virus attacks its human host by attaching its surface proteins onto the receptors of healthy cells called ACE2 receptors. When the virus is bound to the ACE2 receptor, its RNA is able to enter the cell and make copies of itself. As a result, the virus can replicate itself rapidly once inside the body of its host (Patel, 2020). However, the viral particle is surrounded by a layer of fat (Patel, 2020), which can easily be dissolved with soap or a solution with a concentration of alcohol 60 percent or higher, leaving the virus vulnerable (Patel, 2020). Thus, soap and most sanitizers are effective in cleaning hands and surfaces by killing the virus.

\section{Background and Methods}

To formulate my own opinions about the preparation of future pandemics, I analyzed the successes and failures of the current pandemic while noting the virus's biology and molecular structure. To begin my research, I used articles from PubMed to understand the virus on a molecular level. I narrowed down my research by using keywords such as "lifespan of virus" and "virus transmission." To review previous pandemic measures, I used the official Centers for 
Disease Control and Prevention (CDC) website and the World Health Organization (WHO) website. These websites were the most helpful and most reliable, as they were written by multiple health officials and experts. Only reputable articles and other sources were used to ensure the accuracy of the information being researched. I also aimed to use articles that were most recently published so that the information would be the most updated and relevant to the future.

In addition, I analyzed the effectiveness of each preventative measure taken by the United States government during the current pandemic using the CDC website. Using my previous knowledge on the virus's biology and its transmissibility, I modified these preventative measures to better account for factors that were unknown to the general public prior to the pandemic.

Furthermore, I used keywords such as "coronavirus vaccine" and "coronavirus medicine" to research the existing treatments for the virus, as well as potential treatments. From these, I made and modified recommendations that would best aid the general public in future pandemics.

I also made several new recommendations of my own, including an improved health education program for children and teens in order to educate more of the general public on the virus and to avoid misinformation. Such recommendations and conclusions from my research are based on my personal opinion and my personal priorities in future pandemics, so several aspects that I have included may differ for others who aspire to replicate this project.

\section{Possible Origins of the Virus}

In order to adequately prepare for the next pandemic, the origins of the virus must be analyzed and acknowledged. There are several speculations about the possible origins of the virus, some more plausible than others. Initially, it was assumed that COVID-19 began infecting humans after being transmitted directly from bats (Hinshaw et al., 2020). However, given that bats are not a native species in Wuhan, China, it is more likely that the virus was transmitted from bats through an intermediate host, and then onto humans. Another popular theory is that the virus was deliberately manufactured in a lab (Hinshaw et al., 2020). This idea is highly unlikely, because the structure of the virus shows that it occurs naturally rather than artificially. Whatever the true origin of the virus may be, it is important that scientists research and understand the patterns of the virus to prevent another pandemic from spiraling out of control in the future.

\section{An Analysis of Preventative Measures Enacted by the United States During the Initial Stages of the Pandemic}

During the beginning of the COVID-19 pandemic, the United States government was quick to announce a national health emergency. The CDC even recommended gatherings of fifty people or less (Bowman, 2020). Yet, by March 26, 2020, the United States led the world in COVID-19 cases, with at least 81,000 confirmed infections (McPhillips \& Gan, 2021). Given the rapid exponential rise in cases in the United States, it is clear that these initial guidelines were not as effective as intended. In addition, several states faced COVID-19 test shortages months after the first outbreak in the United States (Ehrlich, 2020). Because of the lack of adequate testing, many states suffered from the inability to track cases (Ehrlich, 2020), specifically asymptomatic cases, which ultimately contributed to the country's overall exponential growth in COVID-19 cases (Anderson, 2021). The initial lack of masks and drugs in the United States also meant that some hospitals were unable to safely and effectively treat their patients, which posed a serious concern for Americans nationwide. Thus, in preparation for the next pandemic, the United States must have adequate resources, such as PCR tests, hospital equipment, and simple hygiene products (sanitizer, masks, and gloves).

\section{Transmission of the Virus}


It is now known that COVID-19 is a highly infectious respiratory disease that can be transmitted through the air when an infected individual talks, sneezes, or coughs (Stadnytskyi et al., 2020). When an infected individual speaks, respiratory droplets, which can be anywhere from 1 micrometer to 500 micrometers in diameter, are generated (Stadnytskyi et al., 2020). In infected individuals, these respiratory droplets carry high amounts of COVID-19 particles, which makes speaking a likely mode of disease transmission (Stadnytskyi et al., 2020). These droplets can remain in the air for several hours, and may even travel farther than 6 feet under windy conditions (Feng et al., 2020).

During the beginning of the pandemic, there was uncertainty about the likelihood of viral transmission via hard surfaces, which contributed to the shortages in rubbing alcohol and sanitizer in early 2020. However, the CDC has confirmed that although there is a possibility of viral transmission through surfaces, this risk is low and is not the main mode of COVID-19 transmission (CDC, 2021). Regardless, as a precaution for the next pandemic, highlytouched surfaces such as door knobs and desks should still be regularly sanitized in both public spaces and homes. In addition, standard methods to maintain good hygiene should still be practiced, which include washing hands frequently with soap and water for at least 20 seconds (CDC, 2021). Individuals should also avoid touching their faces as much as possible to prevent the entry of germs and virus particles to their bodies.

\section{Recommendations for Travel, Masks, Vaccines, Distancing}

Since it is now known how the virus works and is transmitted, the recommendations for travel, mask-wearing, and vaccines can be adjusted and improved accordingly. Because vaccines are not cures for diseases like COVID-19, it is important that effective prevention methods are put in place as soon as a similar pandemic occurs. Travel to and from outbreak epicenters and areas with high infection rates and cases should be restricted to essential travel only as soon as possible to avoid an uncontrollable increase in cases. Those arriving in the United States from these COVID-19 "hot-spots" should follow a strict 14-day quarantine, during which they will have no face-to-face contact with any other individual (CDC, 2020). After the 14 days, if the individual tests negative for the virus via a PCR test, the quarantine may be ended. Otherwise, it is recommended that they continue the isolation until they are cleared of the virus (CDC, 2020). In the event of a similar pandemic, mask mandates should be put into place immediately, because the virus spreads mainly through the air and through face-to-face interactions (CDC, 2020). Masks should cover the chin fully and be worn above the nose to prevent as many particles from entering or exiting the mouth and nose as possible (CDC, 2020). Even though viral particles may travel farther than 6 feet under windy conditions (Feng et al., 2020), if masks are worn correctly, then the risk of exposure to the virus is lowered. In the beginning of the COVID19 pandemic, the United States experienced a mask shortage during which the CDC recommended cloth masks for the public in order to reserve surgical masks for healthcare workers. However, the United States should aim to supply hospitals with ample surgical masks so that everyone, regardless of occupation, is able to protect themselves as much as possible. To avoid a mask shortage, funds should be redirected to suppliers and manufacturers in order to ensure that enough masks are being produced.

In addition, it may be necessary to implement strict lockdowns in states with high transmission rates. In New Zealand, strict lockdown protocols were quickly put in place on March 26th, 2020, following the first few cases in the country (Baker et al., 2020). The lockdown included closing public spaces, implementing a stay-at-home policy and travel bans, and having extensive contact tracing. Within 5 weeks, New Zealand saw a continuous decline in cases (Baker et al., 2020). By May of 2020, they successfully eradicated the virus (Baker et al., 2020). Even though New Zealand wasn't able to stay free of the virus forever, the country still managed to flatten the curve. New Zealand's successful eradication of the virus is an indicator that strict lockdown measures are effective and can be done, which is why the United States should attempt such measures for the next pandemic (Baker et al., 2020).

\section{Education for Children and Teens}


It is important to reduce and prevent misinformation should another similar pandemic occur (WHO, 2021). Educating people of different age groups and occupations will be effective to address their unique situations while providing them with honest research findings. Public school systems should implement an informative but entertaining health class for elementary school students. The goal of this health class is not to instill fear or panic in the children, but rather to expose them to the basics of the virus, how it is transmitted, and how they can better protect themselves. COVID-19 education should be incorporated into the science classes (for high school students, biology classes) of middle and high school students. Ultimately, the media should not be an individual's only source of information; the media is often biased and may contain misinformation.

\section{Drugs and Therapies}

During the beginning of the pandemic, there were several speculations about the usage and efficacy of hydroxychloroquine due to the spread of misinformation. However, in June of 2020, the FDA warned against using hydroxychloroquine, as it could cause serious liver and kidney injuries amongst several other serious side effects. As of August 2021, there is one drug that has been officially approved by the FDA to treat COVID-19 (NIH, 2021); several other potential drugs and therapies are still undergoing further research. The drug approved by the FDA is called remdesivir, an antiviral drug that can be used to treat adults and children age 12 and older. By interfering with the enzymes that the virus uses to replicate RNA, remdesivir is able to prevent the virus from making copies of itself. It is administered intravenously once daily for around 5-10 days (NIH, 2020). Although the drug has some side effects that include nausea, soreness, chills, and cough, it has been proven to be effective against COVID-19. The National Institute of Health (NIH) reports a median recovery time of 10 days for patients using remdesivir, as opposed to the 15-day recovery for patients receiving the placebo $(\mathrm{NIH}, 2020)$. Some antiviral medicines can limit the production of new viruses in host cells, but don't directly kill the virus, making it a promising alternative drug. In addition, researchers are currently looking into monoclonal antibodies, which are aimed to prevent the COVID-19 virus from attaching to the body's receptors (FDA, 2021). These types of medicines are not yet guaranteed to work, as they are still being researched, but should be kept open as possible forms of treatment in the future (DeSimone, 2021). Patients that are considering one or more of these therapies and/or drugs should discuss their options with their doctors to ensure that they receive the options that are right for them. If a patient has an adverse reaction to any drug, they should seek help immediately.

If the patient is unable to obtain the necessary drugs, they should begin recovery at home if their case is mild. The patient's symptoms may be alleviated with rest, sufficient water, and fever-reducing medicine. However, if the patient is having trouble breathing or is having a secondary health issue, they should visit the hospital immediately. For these more severe cases, supplemental oxygen and ventilators may be used.

Everyone who is eligible for the vaccine should aim to be fully vaccinated. Though fully vaccinated individuals can still contract COVID-19, the chance of infection is significantly reduced. In addition, there is also a lower chance that their case will be severe and/or require hospitalization than without the vaccine. However, individuals that are allergic to components found in the vaccine or have had significant adverse reactions to the first dose should not continue with their vaccination. Instead, they should continue practicing safe preventative measures that include social distancing, wearing masks when around others, and limiting travel as much as possible.

\section{Conclusion}

It is essential that we learn from the mistakes made from this current pandemic in order to prevent them during future pandemics; people worldwide have suffered from both direct and indirect consequences of the pandemic. It is also important that health officials around the world are transparent with their country's findings, so that citizens are able to better understand the situation and protect themselves. The United States continues to suffer from the spread of 
misinformation (WHO, 2021), which is why Americans are encouraged to thoroughly conduct their own research using trusted sources instead of relying solely on the media.

In conclusion, the following recommendations (in no specific order) should be prioritized for future pandemics: a stricter and more immediate lockdown response of an area when the first few cases are detected; the redistribution of funds to support the manufacturing of various resources, such as masks, gloves, sanitizers, drugs, and vaccines; effective education programs for individuals of different ages to avoid misinformation and confusion about the pandemic; a mask mandate for all who reside in states with detected cases; and stricter social distancing protocols to avoid face-to-face transmission of the virus.

It is near impossible to fully eradicate a disease like COVID-19, but with better knowledge and preparation, more lives can be saved with less serious consequences.

\section{Acknowledgments}

I would like to thank Dr. Reto Asmis and Mr. Mark Crowder for their help with my research and the formulation of my project.

\section{References}

Anderson, M. (2021, May 11). Asymptomatic coronavirus infections contribute to over $50 \%$ of spread, according to UChicago study. UChicago Medicine. https://www.uchicagomedicine.org/forefront/coronavirus-disease-covid19/asymptomatic-coronavirus-infections-contribute-to-over-50-percent-of-spread

Baker, M. G., Wilson, N., \& Anglemyer, A. (2020). Successful Elimination of Covid-19 Transmission in New Zealand. New England Journal of Medicine, 383(8), e56. https://doi.org/10.1056/nejmc2025203

Bowman, E. (2020, March 15). CDC Recommends Against Gatherings Of 50 Or More. NPR. https://www.npr.org/2020/03/15/816245252/cdc-recommends-suspending-gatherings-of-50-or-more-people-for-thenext-8-weeks

CDC Museum COVID-19 Timeline. (2021). Centers for Disease Control and Prevention. https://www.cdc.gov/museum/timeline/covid19.html

Coronavirus (COVID-19) Update: FDA Authorizes Additional Monoclonal Antibody for Treatment of COVID-19. (2021, May 26). U.S. Food and Drug Administration. https://www.fda.gov/news-events/press-announcements/coronavirus-covid-19-update-fda-authorizes-additional-monoclonal-antibody-treatment-covid-19

COVID-19 and Your Health. (2020, February 11). Centers for Disease Control and Prevention. https://www.cdc.gov/coronavirus/2019-ncov/if-you-are-sick/quarantine.html

COVID-19 and Your Health. (2020b, February 11). Centers for Disease Control and Prevention. https://www.cdc.gov/coronavirus/2019-ncov/prevent-getting-sick/about-face-coverings.html

COVID-19 (coronavirus) drugs: Are there any that work? (2021, August 20). Mayo Clinic. https://www.mayoclinic.org/diseases-conditions/coronavirus/expert-answers/coronavirus-drugs/faq-20485627

Education: From disruption to recovery. (2021, August 5). UNESCO. https://en.unesco.org/covid19/educationresponse

Ehrlich, J. C. (2020, July 19). NIH director says coronavirus testing delays are "too long" as country tries to control virus - CNNPolitics. CNN. https://edition.cnn.com/2020/07/19/politics/nih-director-coronavirus-testing/index.html 
Feng, Y., Marchal, T., Sperry, T., \& Yi, H. (2020). Influence of wind and relative humidity on the social distancing effectiveness to prevent COVID-19 airborne transmission: A numerical study. Journal of aerosol science, 147, 105585. https://doi.org/10.1016/j.jaerosci.2020.105585

Fighting misinformation in the time of COVID-19, one click at a time. (2021, April 27). World Health Organization. https://www.who.int/news-room/feature-stories/detail/fighting-misinformation-in-the-time-of-covid-19-one-click-ata-time

Final report confirms remdesivir benefits for COVID-19. (2020, October 27). National Institutes of Health (NIH). https://www.nih.gov/news-events/nih-research-matters/final-report-confirms-remdesivir-benefits-covid-19

Gan, D. C. M. A. N. (2021, July 8). Global Covid-19 death toll surpasses 4 million. CNN. https://edition.cnn.com/2021/07/07/world/global-covid-death-toll-4-million-intl-hnk/index.html

Hinshaw, D., Page, J., \& McKay, B. (2021, July 23). What We Know About the Origins of Covid-19. WSJ. https://www.wsj.com/articles/what-we-know-about-the-origins-of-covid-19-11624699801

Patel, N. V. (2020, April 15). How does the coronavirus work? MIT Technology Review. https://www.technologyreview.com/2020/04/15/999476/explainer-how-does-the-coronavirus-work/

Stadnytskyi, V., Bax, C. E., Bax, A., \& Anfinrud, P. (2020). The airborne lifetime of small speech droplets and their potential importance in SARS-CoV-2 transmission. Proceedings of the National Academy of Sciences, 117(22), 11875-11877. https://doi.org/10.1073/pnas.2006874117 